\title{
A Hybrid Differential Evolution and Back-Propagation Algorithm for Feedforward Neural Network Training
}

\author{
Partha Pratim Sarangi \\ Computer Science and Engineering \\ Seemanta Engineering College \\ Mayurbhanj, Odisha, INDIA
}

\author{
Abhimanyu Sahu \\ Computer Science and Engineering \\ Seemanta Engineering College \\ Mayurbhanj, Odisha, INDIA
}

\author{
Madhumita Panda \\ Computer Science and Engineering \\ Seemanta Engineering College \\ Mayurbhanj, Odisha, INDIA
}

\begin{abstract}
In this study a hybrid differential evolution-back-propagation algorithm to optimize the weights of feedforward neural network is proposed.The hybrid algorithm can achieve faster convergence speed with higher accuracy. The proposed hybrid algorithm combining differential evolution (DE) and back-propagation (BP) algorithm is referred to as DE-BP algorithm to train the weights of the feed-forward neural (FNN) network by exploiting global searching feature of the DE evolutionary algorithm and strong local searching ability of the BP algorithm. The DE has faster exploration property during initial stage of global search for the expense of convergence speed. On the contrary, the problem of random initialization of weights may lead to getting stuck at local minima of the gradient based BP algorithm. In the proposed hybrid algorithm, initially we use global searching ability of the DE to move towards global optimal solution in the search space for few generations by selecting good starting weights and then precise local gradient searching of the BP in that region to converge to the optimal solution with increased speed of convergence. The performance of proposed DE-BP is investigated on a couple of public domain datasets, the experimental results are compared with the $\mathrm{BP}$ algorithm, the DE evolutionary training algorithm and a hybrid real-coded GA with back-propagation (GA-BP) algorithm . The results show that the proposed hybrid DE-BP algorithm produce promising results in comparison with other training algorithms.
\end{abstract}

\section{General Terms:}

Pattern Recognition, Evolutionary Algorithms

\section{Keywords:}

Differential Evolution, Feedforward Neural Network, Backpropagation algorithm, Real-Coded Genetic algorithms

\section{INTRODUCTION}

In the past few years, pattern classification using feed-forward neural networks (FNN), in specific, multilayer perceptron (MLP) is considered as promising neural network model [2] due to its capability to classify the real world complex problem without prior knowledge on the problem domain. In classification, first the network is trained on a set of paired data from the dataset to evolve a set of free network parameters that is, selection of optimal weights of the network weights and second, then the network is ready to test a new set of data [5].Training the weights of the multilayer perceptron can be considered as an optimization problem in which the network weights are optimized. Now a days, several algorithms have been used to train the neural networks, out of these some are gradient based and others are evolutionary algorithms. Among them the most popular and widely used training algorithm is the backpropagation training algorithm [10, 11] , which is a gradient based approach. However, there exist some inherent problems in the backpropagation algorithm. First, the convergence speed of the backpropagation is slow for training a large size network. Second, for complex non-linearly separable problems or complex function approximation the back-propagation algorithm easily gets stuck in local minima. Third, the training performance is very sensitive to the learning rate and momentum parameters of the algorithm. Fourth, the proper decision boundary depends on the sequence of the input data of the training set. However Curry and Morgan [6] pointed out that $\mathrm{BP}$ and gradient techniques may not always produce the best and fastest way to train neural networks.

In the recent years, evolutionary algorithms play a predominant role to improve learning process of feedforward neural networks. Evolutionary algorithms (EAs) are global search techniques adopting the principle of natural biological evolution and/or the social behavior of species. Unlike gradient descent techniques these algorithms start with a population of candidate solutions of the problem, which makes them able to escape from local optima. To overcome the disadvantages inherent to BP, global search based optimization algorithms are being used as learning method for feed-forward neural networks. Many researchers have introduced evolutionary algorithms to optimize the neural networks weights globally in order to avoid the local minima that so often appear in complex problems. Montana and Davis [7] showed how any neural network can be rewritten as a type of genetic algorithm. Whitley [15] attempted successfully to train feed-forward neural networks using genetic algorithms. Sexton et al. compared back-propagation with a simulated annealing [8] genetic algorithm [9, 10] for neural networks training. X. Yao [11] gave new dimension to neural networks. J. D. Schaffer et al.[17] wrote a survey on combinations of genetic algorithms and neural networks. The performance of back-propagation algorithm compared with binary and real-coded genetic algorithms for training multi-layer perceptron has been referred in [2, 3]. Iloren et al. [1] compared back-propagation with differential evolution for neural networks training. Adam Slowik et al. [23] used differential evolution as global search method to train MLP for classification of 
parity-p problem. Some of the previous works using particle swarm optimization in [18, 19, 20, 21]for training feed-forward neural networks.

This global search ability of EAs improves the performance of feedforward neural networks, at the expense of very high computation complexity. This computational burden includes evolution of each solution i.e. the parameters of the FNN, which requires learning entire training set. One of the possible methods for eliminating this shortcoming is to develop a hybrid algorithm which incorporates the gradient descent learning followed by evolution search.

This paper is motivated by the work of Ilonen et al. presented in [1], in that authors used differential evolution (DE) proposed in [22] to train the weights of FNN. They concluded that the DE algorithm can converge to a global minimum for complex error surface, at the cost of very high computational complexity. In order to speed up the convergence rate and capability of avoiding local optima of DE, we proposed a simple non-Lamarckian hybrid approach by utilizing both evolutionary and gradient information. This hybrid training of FNN using the differential evolution to do global search in the beginning of training, and then the back-propagation algorithm to perform a local search around the global solution in the weight space of the problem to enhance the convergence speed of the training. Moreover, the experiment result shows that DE-BP training algorithm maintain better classification rate all the time without getting stuck at local minimum. Hence in this work, hybrid DE-BP is compared with the conventional back-propagation algorithm and differential evolution based FNN training algorithm in convergence speed and generalization performance using real world bench mark datasets.

The rest of the paper is organized as follows: Section 2 deals with an overview of FNN, BP, GA, and DE algorithms respectively with a special emphasis on their strengths and weaknesses; Section 3 focuses on the detail of proposed algorithm; Section 4 describes simulation of four algorithms on 7 real world datasets with their result analysis; Section 5 provides conclusions about our work and suggestions for future work.

\section{BACKGROUND STUDY}

We use four algorithms for our study to train the weights of the feedforward neural network with two layered structures:the backpropagation algorithm, the differential algorithm, the hybrid genetic algorithms and Backpropagation, and the hybrid differential evolution and backpropagation. We briefly describe them in the following paragraphs.

\subsection{Artificial Neural Networks}

An artificial neural network (ANN) is a well-known computational model which maps input patterns from measurement space into predefined classes in the decision space. The feedforward neural networks (FNN) are widely used model for pattern classification and approximate continuous functions. The most popular FNN model is multilayer perceptron (MLP) [14] comprises a set of sensory nodes that constitute the input layer, one or more hidden layer of processing nodes, and an output layer of processing nodes. Fig.(1)shows three layers MLP in which neurons are structured into ordered layers, and weighted connections are allowed only between adjacent layers units or neurons. The number of nodes in input layer represents the coordinates of the input vector and the number of nodes in the output layer corresponds to the number of output classes. However the nodes in the hidden layer have decided optimally by trial and error basis in the experiments, these numbers of nodes

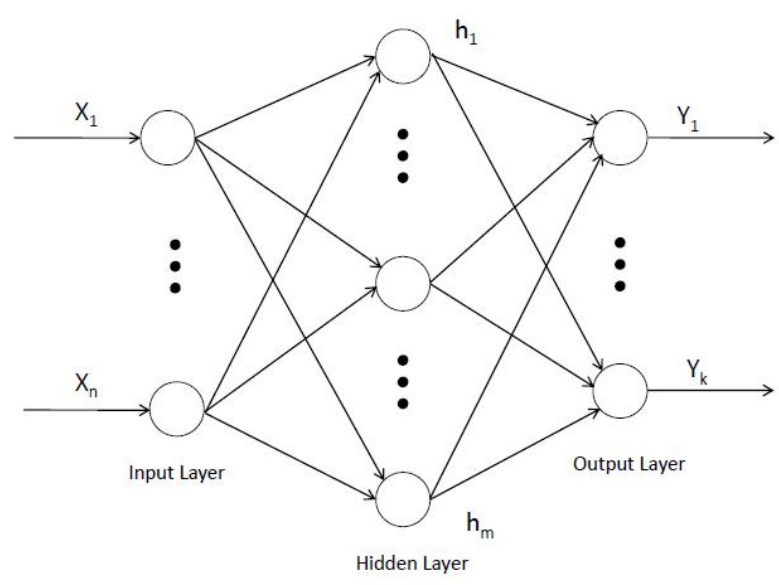

Fig. 1. Schematic representation of multilayer perceptron

vary from problem to problem. The network weights comprise both connection weights and bias term for each unit. The process of updating network weights is called training or learning of the neural network. For classification application supervised learning process is used, with a set of input patterns and desired outputs are used for training. The input and output of the network are both real vectors in our case.

In the supervised training change of network weights depends upon the instantaneous error between actual and expected outputs of the network. Defining the error function by the network actual output with M neurons, that is:

$$
e=\sum_{k=1}^{M}\left(d_{k}-y_{k}\right)^{2}
$$

where $d_{k}$ and $y_{k}$ are respectively the $k^{t h}$ component of the expected and actual output vector. This error term can be just for one single pattern or for a set of patterns depends on on-line or batch learning. In the experiment, we use batch learning there mean square error is obtained by summing individual errors over all training patterns $\mathrm{N}$.

$$
E_{M S E}=\frac{1}{N . M} \sum_{i=1}^{N} e_{i}=\frac{1}{N . M} \sum_{i=1}^{N} \sum_{j=1}^{M}\left(d_{i j}-y_{i j}\right)^{2}
$$

\subsection{A brief introduction of BP}

The BP algorithm is simply a gradient descent method [12,13] designed to minimize the total error of the output computed by equation(2) of the network in Fig.(1) using all training patterns. The total input $x_{j}^{h}$ received by neuron $\mathrm{j}$, in layer $\mathrm{h}$, is defined as

$$
x_{j}^{h}=\sum y_{i}^{h-1} w_{j, i}^{h-1}+b_{j}^{h-1}
$$

Where $y_{i}^{h-1}$ is the state of the $i^{t h}$ neuron in the preceding h-1th layer, $w_{j, i}^{h-1}$ is the weight of the connection from the $i^{t h}$ neuron in the layer $\mathrm{h}$ to the $j^{\text {th }}$ neuron in the layer h- 1 and $b_{j}^{h-1}$ is the threshold of the $j^{t h}$ neuron in layer h-1. Threshold may be added by giving the unit $\mathrm{j}$ in layer $\mathrm{h}$ an extra input line with a fixed activity level of 1 and a weight of $b_{j}^{h}$. 
The output of a $j^{t h}$ neuron, $x_{j}$ in any layer other than the input layer $(h>0)$, is a parameter to a non-linear activation function of its total input and we use hyperbolic tangent activation function, defined as,

$$
y_{j}^{h}=\frac{1-e^{-x_{j}^{h}}}{1-e^{-x_{j}^{h}}}
$$

All neurons within a layer, other than an input layer, have their states set by (1) and (2) in parallel, while different layers have their states set sequentially one after another layers forward manner until the states of the neurons in the output layer $\mathrm{H}$ are determined. The learning procedure determines the internal free parameters of the hidden units based on its knowledge of its inputs and the desired outputs. Hence learning consists of searching a very large parameter set and therefore is usually rather slow.

The least mean square (LMS) error in output vectors, for a given network is defined as,

$$
E(w)=\frac{1}{2} \sum\left(y_{j, c}^{H}(w)-d_{j, c}\right)^{2}
$$

where $y_{j, c}^{H}(w)$ is the state obtained for output node $\mathrm{j}$ in output layer $\mathrm{H}$ and $d_{j, c}$ is its desired state specified in the supervised learning. One method of minimization of $\mathrm{E}(\mathrm{w})$ is to apply the method of gradient descent by starting with any set of weights and repeatedly updating each weight by an amount

$$
\Delta w_{j i}^{h}(n+1)=\eta \Delta w_{j i}^{h}(n-1)+\alpha \Delta w_{j i}^{h}(n-1)+h d e c . w_{j i}^{h}(n-1)
$$

where the positive constant $0<\eta<1$ controls the descent, $0<\alpha<1$ is the damping coefficient or momentum controls acceleration, hdec is the percentage decay coefficient and $n$ is the number of epoch currently in progress. Using a decay factor $0.01>h d e c>0$ enables only those weights doing useful works in reducing the error to survive and hence improve the generalization capabilities of the network.

\subsection{A brief introduction of GA}

A genetic algorithms (GA), one of the evolutionary algorithms is a heuristic stochastic global search optimization technique that mimics the process of natural evolution. Like other evolutionary algorithms, GA is a population-based iterative search algorithm which searches from one population to another, focusing on the area of the best solution so far, while continuously searching the solution space generation wise. To achieve best solution, the GA applies stochastic operators such as selection, crossover and mutation. The major steps of GA include: encoding, initialization of the population, fitness evaluation, selection, crossover and mutation. In this work we use real valued chromosome instead of binary representation of the weights.

\subsubsection{Pseudo-code of Genetic Algorithms}

(1) $\mathrm{i}=0$

(2) Initialize population

(3) fitness evaluation for initial population

(4) while termination criteria not satisfied $\neq$ true \{

Selection

Crossover

Mutation

Fitness evaluation for new population

$$
i=i+1
$$$$
\text { \} }
$$

end while

\subsection{A brief introduction of $\mathrm{DE}$}

Differential evolution (DE), proposed by Storn and Price in [23], is an efficient and simple evolutionary algorithm for real parameter optimization problems. In the last few years, the DE algorithm has been successfully applied to many science and engineering applications. Similar to other EAs, DE is a population based stochastic optimization method. Like other evolutionary algorithms, DE commences with a population of constant size of NP individual candidate solutions, where NP is the population size. Each member is a D-dimensional real-parameters vector representing a point in the solution space $\mathrm{S}$. The new candidate solutions of same population size are obtained generation by generation in the solution space. The subsequent generations are denoted by $G=0,1, \operatorname{Gmax}$. However the vectors are changed over generation wise by a special kind of differential operator instead of classical crossover and mutation operators of GA.

The number of connection weights in the network is the parameters of a candidate solution. For L number of layers, number of parameters is.

$$
P=\sum_{i=1}^{L-1} p_{i+1}\left(p_{i+1}\right)
$$

Each parameter is represented a real value in a range of $(-1,1)$.

\subsubsection{Pseudo-code of DE Algorithm}

(1) Initialize the generation $G=0$ and a population PG of NP individuals randomly in the uniformly distributed range $\left[X_{\max }, X_{\min }\right]$

(2) While $G<G \max$

$\{$ for $i=1$ to NP do

$\left\{\right.$ Randomly generate three integer numbers $r_{1}, r_{2}$, and $r_{3}$ from $[1, N P]$, where $r_{1} \neq r_{2} \neq r_{3} \neq i$

$\{$ for $j=1$ to D do mutation :Generate $i^{\text {th }}$ donor vector $V_{i}$,

$$
v_{i, j}=x_{r_{1}, j}+F *\left(x_{r_{2}, j}-x_{r_{3}, j}\right)
$$

Rearrangement :keep each parameters of donor vector in the range $\left[X_{\min }, X_{\max }\right]$. crosover :Generate $i^{t h}$ trial vector $U_{i}$ If rand $(0,1)<C R$, then $u_{i, j}=v_{i, j}$ Else \} end

$$
u_{i, j}=x_{i, j}
$$

Selection and replacement :

If individual $u_{i}$ fitness is better than individual $x_{i}$ then Replace individual $x_{i}$ by $u_{i}$ individual \}

\} end

end

The $\mathrm{F}$ parameter scales the difference of two vectors and the scaled difference is added to the third vector to obtain the donor vector. The CR parameter represents the crossover rate. The global optimal searching capability and the convergence speed are affected by NP, F, and CR parameters. The parameter values $F$ and $\mathrm{CR}$ are selected by the user in the range $(0,2]$ and $[0,1)$. 


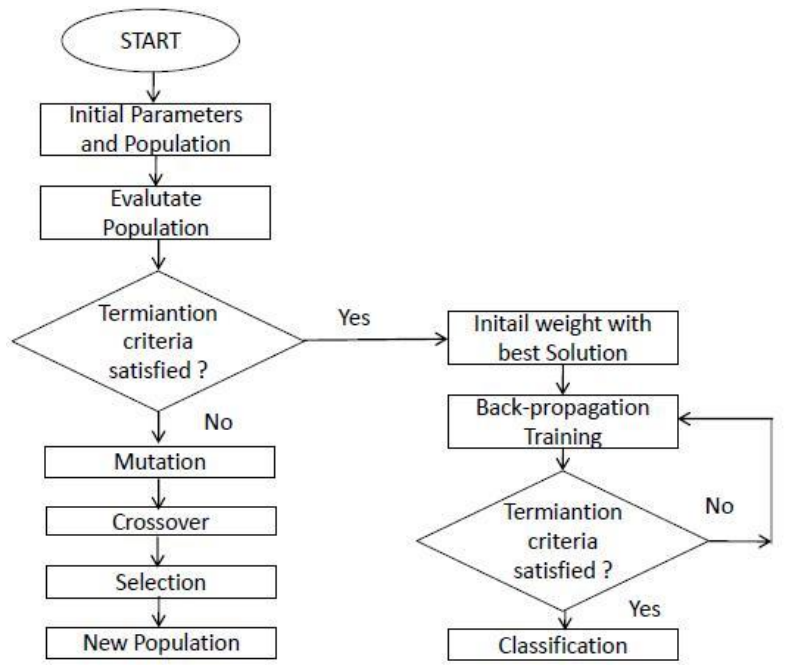

Fig. 2. Framework of hybrid algorithm for classification

\subsection{Hybrid Algorithms}

To overcome the local minima problem of BP due to initial random weight parameters of the network a number of evolutionary algorithms has been tried by many researchers, which improve the performance of the classification at the cost of more execution time. In this work we hybridized two algorithms, combining global search evolutionary algorithm and local search gradient algorithm that overcomes the local minima problem with high generalization and fast convergence speed. We tried two hybrid algorithms: a GA with the $\mathrm{BP}(\mathrm{GA}-\mathrm{BP})$ algorithm and a $\mathrm{DE}$ with the $\mathrm{BP}$ proposed (DE-BP) algorithm.

\section{PROPOSED DE-BP ALGORITHM}

The hybrid DE-BP is a training algorithm combining the DE algorithm with the BP. Similar to the GA, the DE is an evolutionary population based global optimization algorithm, which has strong ability to explore the entire search Space. This algorithm has a disadvantage that the search around the global optimum solution is very slow. In contrary, the BP has precise and fast local searching ability to explore locally the optimum result, but it suffers to find global optimum result in complex search space.

By combining the evolutionary DE and the gradient based BP algorithm, a new algorithm referred to as hybrid DE-BP algorithm, illustrated in Fig. (2). The proposed hybrid algorithm has two stages: first one a global search phase, the FNN is trained using the $\mathrm{DE}$ algorithm for few pre-defined generations or training error is smaller than some predefined value, then training process switched to second phase for searching locally using a deterministic method such as the back-propagation algorithm. In this work, it realized DE-BP hybrid training algorithm as a successful alternative approach to BP algorithm.

The DE-BP algorithm's searching process is started from initializing a group of random target vectors as population. First, all the target vectors are updated using mutation operator and produce donor vectors. In the crossover, for each donor vector and for all components check if rand $(0,1) ; \mathrm{CR}$ then change the donor vector component else that component will remain in the donor vector and finally trial vectors are generated. Then selection offspring by fitness value of each trial vector is compared with the correspond-
Table 1. Summary of used datasets.

\begin{tabular}{lllllc}
\hline Datasets & Instance & $\mathrm{b}$ & $\mathrm{c}$ & $\mathrm{t}$ & class \\
\hline Cancer & 683 & 0 & 9 & 9 & 2 \\
Bupa live & 345 & 0 & 6 & 6 & 2 \\
Diabetes & 768 & 0 & 8 & 8 & 2 \\
iris & 150 & 0 & 4 & 4 & 3 \\
Thyroid & 215 & 0 & 4 & 4 & 3 \\
wine & 178 & 0 & 13 & 13 & 2 \\
Zoo & 101 & 15 & 2 & 17 & 7 \\
\hline
\end{tabular}

ing target vector, best fit vector will be selected as new offspring for next generation and then those new target vectors are used to search the global best position in the solution space. Finally the BP algorithm is used to search around the global optimum. In this way, proposed hybrid algorithm improves the convergence speed than the DE algorithm and less chance to get stuck in local minima like the BP algorithm.

The pseudo code for this hybrid DE-BP algorithm can be summarized as follows:

STEP 1: Initialize the DE parameters.

STEP 2: Initialize the population with real values in the domain $[0,1]$ for each neuron's connection weights and bias to its corresponding gene segments

STEP 3: While new gen. is less than equal to MaxGen.DO\{

STEP 4: The fitness of target vectors are determined by MSE

STEP 5: Sort minimum fitness values.

STEP 6: If first fitness value is less than equal to min. error then select best solution for MLP then goto step. 9

STEP 7: Generate donor vectors of the population using mutation

STEP 8: Generate trail vectors of the population using crossover

STEP 9: Selection of new offspring of the population for new generation

STEP 10: Initialize parameters of back-propagation learning

STEP 11: Initialize weights of the MLP using best solution of RGA

STEP 12: While new epoch is less than equal to MaxEpoch or error converges to Min Error do

STEP 13: Update weights to minimize error using backpropagation with training data

STEP 14: End while

STEP 15: Evaluate performance of classification with test data

STEP 16: End while

\section{EXPERIMENTAL STUDY}

After discussing the algorithms, in this section we present the experiments performed and their results. The benchmark data sets, training methodology and experimental parameters setting are presented in the next subsection 4.1 and 4.2. The analysis of the results is explored in subsection 4.3. 
Table 2. FNN Architecture and patterns distribution of all datasets.

\begin{tabular}{llccc}
\hline Datasets & Architecture & Training & Validation & Test \\
\hline Breast cancer & $9-10-2$ & 274 & 136 & 273 \\
Bupa live & $6-7-2$ & 138 & 69 & 138 \\
Pima diabetes & $8-10-2$ & 304 & 154 & 310 \\
Iris & $4-5-3$ & 60 & 30 & 60 \\
Thyroid & $5-8-3$ & 86 & 43 & 86 \\
Wine & $13-10-3$ & 71 & 36 & 72 \\
Zoo & $16-10-7$ & 40 & 20 & 41 \\
\hline
\end{tabular}

\subsection{Experimental real world datasets}

We use seven datasets from different classes as summarized in table 1 to compare the performances of $\mathrm{BP}$ algorithm, DE based training algorithm, GA-BP algorithm and DE-BP algorithm in optimizing the weights of the FNN. These data sets are the Wisconsin breast cancer, bupa liver diagnoses, pima diabetes, fisher's iris plant, thyroid dysfunction, wine and zoo data sets. Table 1 lists a summary of the used datasets along with following attributes: datasets and the number of instances, the number of binary (b), continuous (c) features in the dataset, the total $(\mathrm{t})$ number of features, and number of classes. The data sets were obtained from the UCI repository [24].

In this paper, we use FNN in particular multi-layer perceptron (MLP) with three layers (input-hidden-output). The number of neurons in the input and output layers of the FNN depends on the features and classes of the concerned data set used in the experiment. The performance of the FNN gets affected by number of neurons in the hidden layer. We adopt trial and error process to decide the number of neurons in the hidden layer by considering the performance of the FNN. Table 2 summarizes the network architecture for each data set.

In the classification problems, the data sets are used to determine the class that a certain input vector belongs to. Each pattern from the training set consists of an input vector and its desired output vector. These input and output vectors are normalized and represented as real vectors. The size of the input and output vectors are depended on number of features and classes present in the data set. When an input vector is assigned to the FNN, the network response is one of the classes associated with the output neuron having greater value.

To evaluate a FNN, each data set splits into three parts: the training patterns, the validation patterns and the test patterns [16]. The first two sets are used for training algorithm and last one is used for testing. The forty percent of the data set for training, twenty percent for the validation and remaining forty percent for testing. A popular and very useful form to use validation set in neural network is early stopping. Indeed the validation set used for testing the performance of the network in the training phase and it is necessary to avoid the overtraining phenomenon. Before partition of the dataset, it is normalized in the range of $[0,1]$. We run all the algorithm of our experiment ten times for every data set and evaluate average performance of the FNN.

The proposed algorithm DE-BP is compared with BP algorithm and $\mathrm{DE}$ based training algorithm, GA-BP algorithm and DE-BP proposed one were implemented and analyzed using matlab.

\subsection{EXPERIMENTAL PREPARATION}

The following steps summarized the preparation of the experiment and configuration parameters of all algorithms.

(1) Before partition of the data set, patterns are normalized

(2) Then partition of the data set randomly into two groups: sixty percent of training and forty percent of test patterns

(3) Again training patterns is partitioned into two groups: forty percent of training and twenty percent of validation

(4) Synaptic weights of the MLP are randomly initialized by real values in a domain $[-1,1]$

(5) Configuration parameters of MLP like learning rate $(\eta)=$ 0.001 , momentum $(\alpha)=0.9$ and decay coefficient $(\mathrm{hdec})=$ 0.0002

(6) Training stops by three ways: (1) The $G L_{5}$ stopping criterion; (2) training error ; 0.001 ; (3) the maximum number of iterations is satisfied

(7) Each candidate solution in the population represents the neural network architecture

(8) The length of the candidate solution is the total number of connection weights of the network

(9) The real-coded GA and DE algorithm use real parameters to represent candidate solutions

(10) Population size is 100 for all three algorithms: DE, GA-BP and DE-BP

(11) DE algorithm use DE/best/1/bin strategy

(12) If the elements of the donor vectors are out of search space then repair operator is used

(13) In DE to increase the potential diversity of the population, a crossover operator is used, binomial crossover has used with Crossover rate: 0.7

(14) Elitism the best solution means lower fitness value is preserved for the next generation

(15) Number of generationsin each experimentfor GA-BP and DE$\mathrm{BP}$ is 100

(16) The GA-BP algorithm uses rank-based selection

(17) The GA-BP algorithm uses arithmetic crossover with crossover rate 0.7

(18) The GA-BP uses non-uniform mutation with mutation rate 0.01

(19) Each classifier repeatedly executes ten times over all datasets

(20) NMSE and accuracy of the classifiers compared in table 3, 4 and 5 .

\subsection{EXPERIMENTAL RESULT ANALYSIS}

Each training algorithm was executed ten times over all datasets mentioned in Table 1and the average accuracy of the classifier (the percentage of samples that it correctly classified) is computed. The results were obtained for each training technique by the optimization of the connection weights of the FNN. The fitness function values (errors) were normalized and average was calculated over total number of training samples. Finally in batch learning mean square error (mse) was obtained that updated the connection weights of the FNN. Moreover the classification rate of the test set obtained in the training of the FNN using different training algorithms, presented in Table 2. Fig. (3) show the graphs comparing the mean square error (mse) and classification rates of the investigated training algorithms over all datasets. The proposed algorithm obtained the best 
Table 3. Average,Standard deviation,min;and max of NMSE and accuracy for all training samples over ten independent runs for $\mathrm{BP}, \mathrm{DE}, \mathrm{GA}-\mathrm{BP}$, and DE-BP

\begin{tabular}{|c|c|c|c|c|c|c|c|}
\hline Dataset & Algorithm & AVG MSE & STD MSE & AVE ACC & STD ACC & MAX ACC & MIN ACC \\
\hline \multirow{4}{*}{ BCW } & $\mathrm{BP}$ & $2.16 \mathrm{E}-02$ & $2.50 \mathrm{E}-03$ & $9.66 \mathrm{E}+01$ & $6.62 \mathrm{E}-01$ & $9.60 \mathrm{E}+01$ & $9.78 \mathrm{E}+01$ \\
\hline & $\mathrm{DE}$ & $9.70 \mathrm{E}-02$ & $2.00 \mathrm{E}-03$ & $9.74 \mathrm{E}+01$ & $6.33 \mathrm{E}-01$ & $9.68 \mathrm{E}+01$ & $9.83 \mathrm{E}+01$ \\
\hline & GA-BP & $2.26 \mathrm{E}-02$ & $1.20 \mathrm{E}-03$ & $9.79 \mathrm{E}+01$ & $5.49 \mathrm{E}-01$ & $9.70 \mathrm{E}+01$ & $9.80 \mathrm{E}+01$ \\
\hline & DE-BP & 2.01E-02 & 1.00E-03 & $9.79 \mathrm{E}+01$ & 5.19E-01 & $9.71 \mathrm{E}+01$ & $9.90 \mathrm{E}+01$ \\
\hline \multirow[t]{4}{*}{ BUPA LIVE } & BP & 8.37E-02 & $1.15 \mathrm{E}-02$ & $6.81 \mathrm{E}+01$ & $3.60 \mathrm{E}+00$ & $6.45 \mathrm{E}+01$ & $7.68 \mathrm{E}+01$ \\
\hline & $\mathrm{DE}$ & 8.61E-02 & $7.00 \mathrm{E}-03$ & $7.19 \mathrm{E}+01$ & $2.43 \mathrm{E}+00$ & $6.76 \mathrm{E}+01$ & $7.44 \mathrm{E}+01$ \\
\hline & GA-BP & $9.49 \mathrm{E}-02$ & $1.74 \mathrm{E}-02$ & $7.32 \mathrm{E}+01$ & $4.27 \mathrm{E}+00$ & $6.74 \mathrm{E}+01$ & $7.87 \mathrm{E}+01$ \\
\hline & DE-BP & 7.07E-02 & 1.15E-03 & $7.49 \mathrm{E}+01$ & $2.24 \mathrm{E}+00$ & $7.10 \mathrm{E}+01$ & $7.98 \mathrm{E}+01$ \\
\hline \multirow[t]{4}{*}{ PIMA } & $\mathrm{BP}$ & 7.22E-02 & $9.30 \mathrm{E}-03$ & $7.29 \mathrm{E}+01$ & $3.46 \mathrm{E}+00$ & $6.66 \mathrm{E}+01$ & $7.79 \mathrm{E}+01$ \\
\hline & $\mathrm{DE}$ & $7.00 \mathrm{E}-02$ & $6.74 \mathrm{E}-03$ & $7.48 \mathrm{E}+01$ & $1.25 \mathrm{E}+00$ & $7.29 \mathrm{E}+01$ & $7.68 \mathrm{E}+01$ \\
\hline & GA-BP & $7.04 \mathrm{E}-02$ & $9.46 \mathrm{E}-03$ & $7.50 \mathrm{E}+01$ & 7.72E-01 & $7.44 \mathrm{E}+01$ & $7.66 \mathrm{E}+01$ \\
\hline & DE-BP & 5.05E-02 & 2.64E-03 & $7.67 \mathrm{E}+01$ & $7.05 \mathrm{E}-01$ & $7.40 \mathrm{E}+01$ & $7.99 \mathrm{E}+01$ \\
\hline \multirow[t]{4}{*}{ IRIS } & $\mathrm{BP}$ & $3.04 \mathrm{E}-02$ & $2.90 \mathrm{E}-03$ & $9.77 \mathrm{E}+01$ & $1.41 \mathrm{E}+00$ & $9.50 \mathrm{E}+01$ & $1.00 \mathrm{E}+02$ \\
\hline & DE & $2.54 \mathrm{E}-02$ & $2.65 \mathrm{E}-03$ & $8.99 \mathrm{E}+01$ & $4.76 \mathrm{E}+00$ & $8.33 \mathrm{E}+01$ & $9.56 \mathrm{E}+01$ \\
\hline & GA-BP & $2.61 \mathrm{E}-02$ & $4.85 \mathrm{E}-03$ & $9.73 \mathrm{E}+01$ & $1.17 \mathrm{E}+00$ & $9.50 \mathrm{E}+01$ & $9.84 \mathrm{E}+01$ \\
\hline & DE-BP & 1.12E-02 & 2.41E-03 & $9.89 \mathrm{E}+01$ & $1.15 \mathrm{E}+00$ & $9.50 \mathrm{E}+01$ & $1.00 \mathrm{E}+02$ \\
\hline \multirow[t]{4}{*}{ THYROID } & BP & $3.97 \mathrm{E}-02$ & $8.70 \mathrm{E}-03$ & $9.31 \mathrm{E}+01$ & $2.77 \mathrm{E}+00$ & $8.72 \mathrm{E}+01$ & $9.65 \mathrm{E}+01$ \\
\hline & $\mathrm{DE}$ & $3.84 \mathrm{E}-03$ & 8.81E-03 & $7.44 \mathrm{E}+01$ & $3.78 \mathrm{E}+00$ & $6.90 \mathrm{E}+01$ & $9.15 E+01$ \\
\hline & GA-BP & $3.75 \mathrm{E}-02$ & $9.94 \mathrm{E}-03$ & $9.49 \mathrm{E}+01$ & $2.58 \mathrm{E}+00$ & $9.19 \mathrm{E}+01$ & $9.88 \mathrm{E}+01$ \\
\hline & DE-BP & 8.26E-02 & $1.23 E-03$ & $9.53 \mathrm{E}+01$ & $2.57 \mathrm{E}+00$ & $9.30 \mathrm{E}+01$ & $9.88 \mathrm{E}+01$ \\
\hline \multirow[t]{4}{*}{ WINE } & BP & $8.46 \mathrm{E}-02$ & $1.34 \mathrm{E}-02$ & $9.74 \mathrm{E}+01$ & $1.20 \mathrm{E}+00$ & $8.72 \mathrm{E}+01$ & $9.65 \mathrm{E}+01$ \\
\hline & $\mathrm{DE}$ & 8.79E-03 & $1.21 \mathrm{E}-04$ & $9.89 \mathrm{E}+01$ & $1.40 \mathrm{E}+00$ & $6.90 \mathrm{E}+01$ & $9.15 \mathrm{E}+01$ \\
\hline & GA-BP & 8.18E-02 & $1.24 \mathrm{E}-02$ & $9.81 \mathrm{E}+01$ & $1.85 \mathrm{E}+00$ & $9.19 \mathrm{E}+01$ & $9.88 \mathrm{E}+01$ \\
\hline & DE-BP & 7.96E-02 & 9.17E-02 & $9.68 \mathrm{E}+01$ & 8.66E-01 & $9.30 \mathrm{E}+01$ & $9.88 \mathrm{E}+01$ \\
\hline \multirow[t]{4}{*}{$\mathrm{ZOO}$} & BP & $2.10 \mathrm{E}-02$ & $2.63 \mathrm{E}-03$ & $9.65 \mathrm{E}+01$ & $2.64 \mathrm{E}+00$ & $9.59 \mathrm{E}+01$ & $9.86 \mathrm{E}+01$ \\
\hline & $\mathrm{DE}$ & $2.00 \mathrm{E}-03$ & $2.34 \mathrm{E}-04$ & $9.68 \mathrm{E}+01$ & $2.43 \mathrm{E}+00$ & $9.44 \mathrm{E}+01$ & $9.81 \mathrm{E}+01$ \\
\hline & GA-BP & $2.16 \mathrm{E}-02$ & 3.39E-03 & $9.60 \mathrm{E}+01$ & $2.46 \mathrm{E}+00$ & $9.45 \mathrm{E}+01$ & $1.00 \mathrm{E}+02$ \\
\hline & DE-BP & 6.53E-02 & 7.34E-03 & $7.02 \mathrm{E}+01$ & $2.41 \mathrm{E}+00$ & $9.73 \mathrm{E}+01$ & $1.00 \mathrm{E}+02$ \\
\hline
\end{tabular}

results in the most dataset where DE based training fails. By taking advantage of global optimization, early stopping, and weight decay proposed algorithm takes less computational time than DE based training algorithm. Similarly, the problem of local minimum of BP algorithm observed many times in running the training algorithm number of times over all datasets. It leads worst performance in the experiments that could be avoided by proposed algorithm. From the experiments, it has revealed that by a large population size the DE training algorithm needs small number of generations to improve its performance. Here we used 50 to 200 population size and achieved better results. In the proposed algorithm population size used 100.Then BP algorithm takes small number of epochs to optimize the connection weights of FNN with less mean square error. In DE based training, maximum generation number was chosen 500. It is analyzed from Table 2 that for almost all datasets the classification rate of the proposed training algorithm outperforms classical back-propagation and DE method. All most all Datasets like bupa, diabetes, iris, thyroid, wine and zoo the proposed algorithm obtained better results than BP and DE method. Moreover in breast cancer dataset DE based training produced best result than proposed training algorithm. Thus it is clearly observed from the table that where $\mathrm{BP}$ and $\mathrm{DE}$ based training not performed well there proposed training algorithm obtained best results. However it has also analyzed from the experiments that DE did not perform well except breast cancer dataset and time needed for convergence always more than BP and proposed algorithm. Hence, no advantage is found in this study to use DE as global optimization training algorithm over conventional BP. But from the results we realized that the proposed algorithm, initially DE evolved a better search space globally in few generations then BP locally searched around the global position to optimize the connection weights of the neural network.

\section{CONCLUSION}

In this paper, we have proposed a hybrid training algorithm for FNN, the hybrid algorithm combining the DE algorithm with backpropagation algorithm, this hybridization exploits the DE algorithm's global search ability and gradient based BP's local search ability. In the experiments, proposed DE-BP algorithm performance has been compared with DE based training algorithm and BP algorithm for FNN training using seven real world standard datasets. Moreover, proposed hybrid algorithm has compared with another hybrid GA-BP algorithm using same datasets. From the experiments, it is revealed that the proposed algorithm outperforms the DE algorithm, the BP algorithm and hybrid GA-BP algorithm. 
$\mathbf{a}$
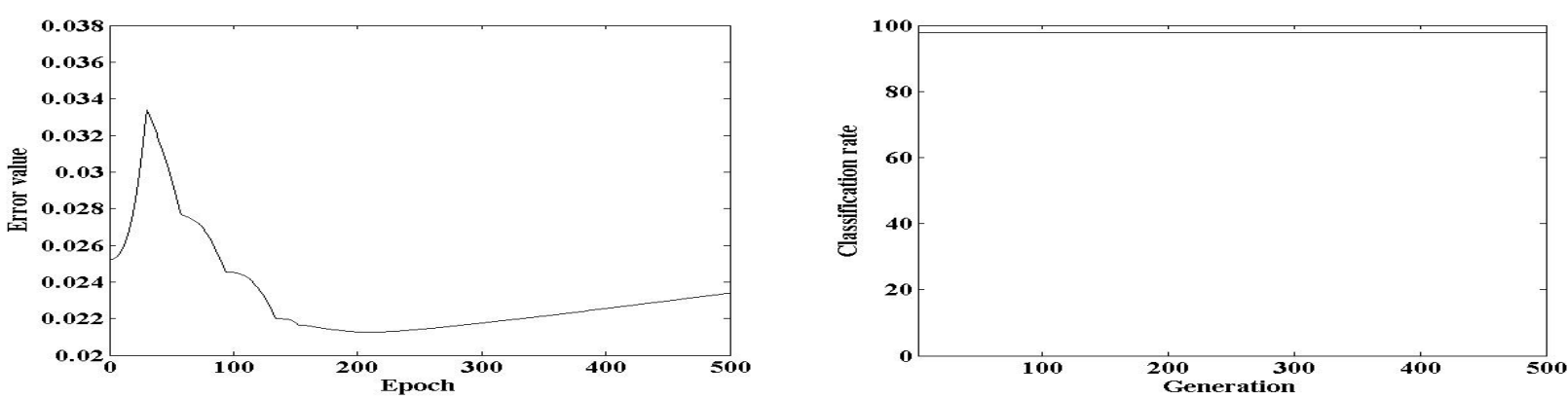

b
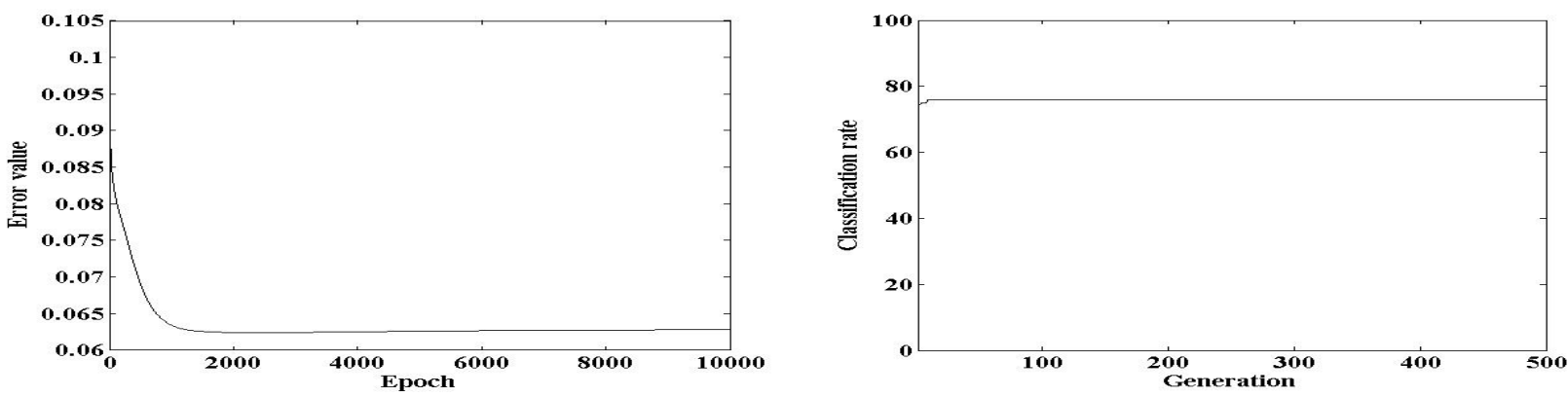

c
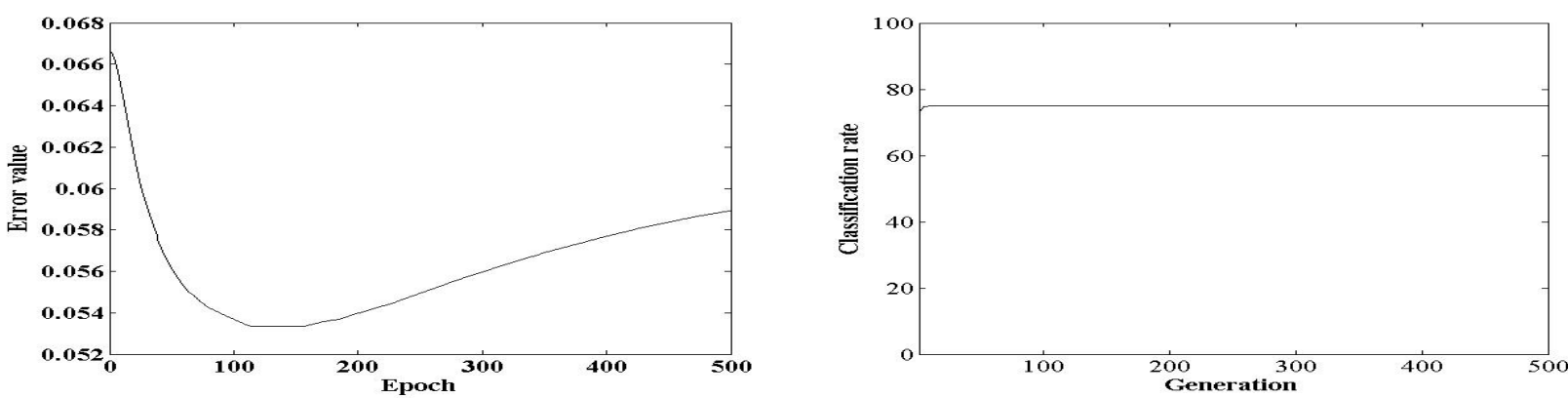

d
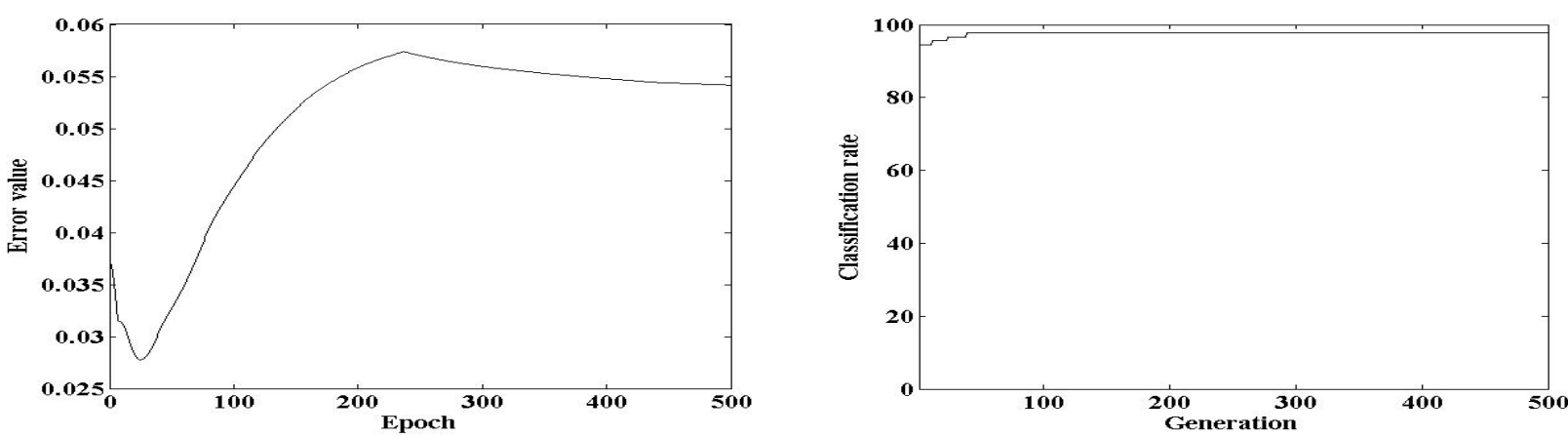

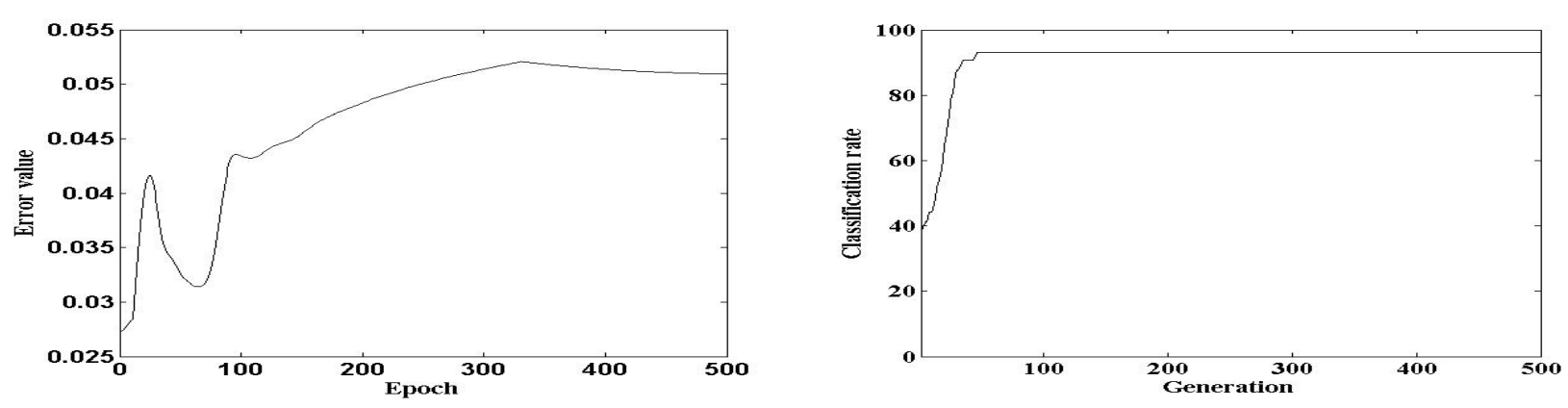

$\mathbf{f}$
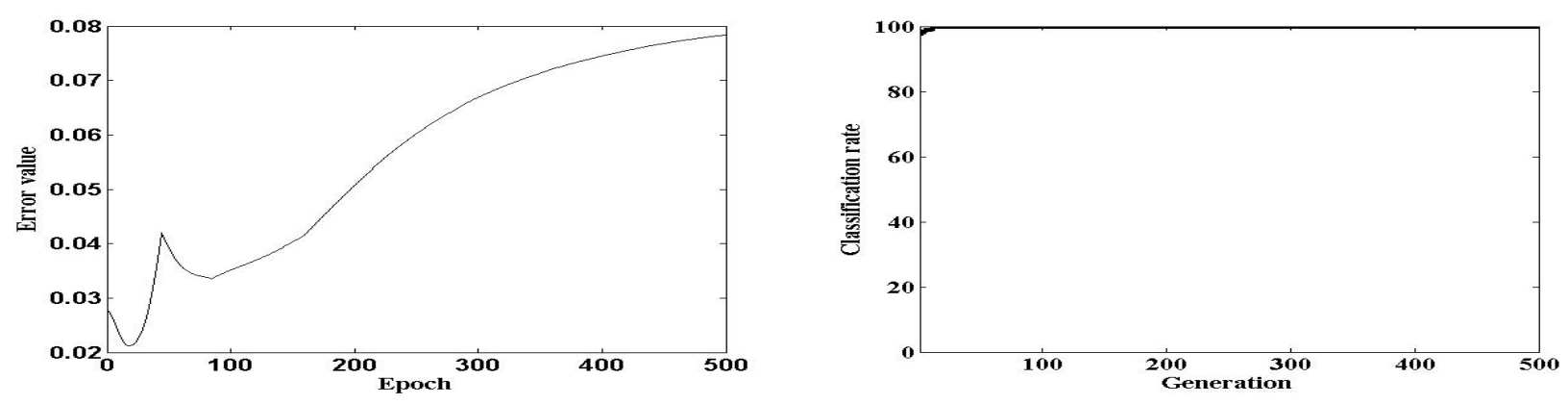

g
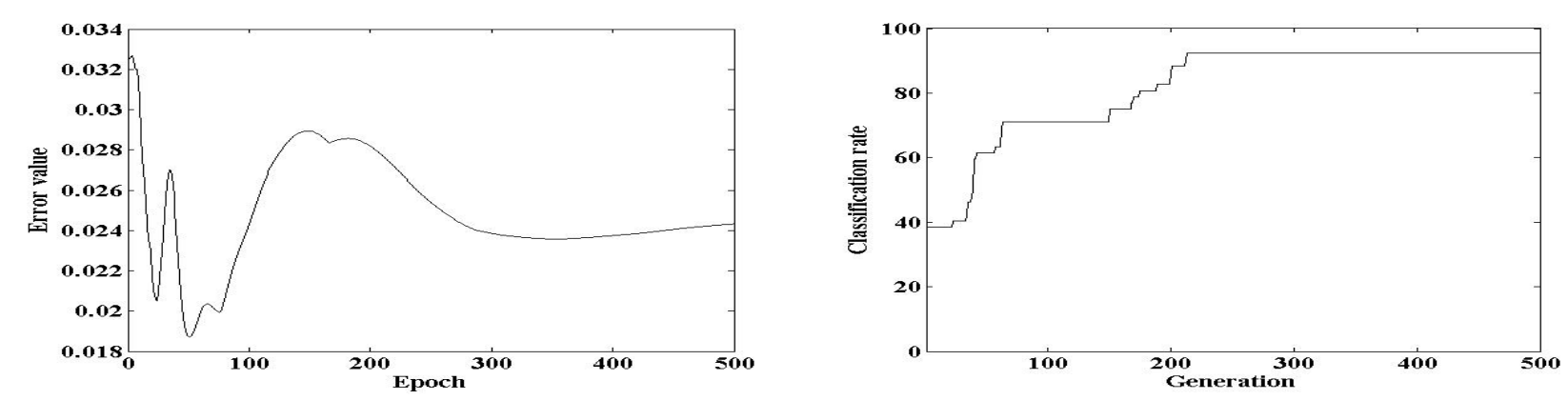

Fig. 3. The training error curves and rate of accuracy curves of De-BP based on average MSE for all training samples over ten independent run in seven datasets (a),(b),(c),(d),(e),(f)and (g) respectively.

The back-propagation algorithm not always performs better and traps in local minima. However, when problems are more complex, back-propagation most often fails because of non-differentiable error functions. The experimental results of the proposed hybrid algorithm improve the training error convergence and classification accuracy than others. Moreover, the proposed algorithm produces higher classification accuracy in less training time than the DE algorithm and BP algorithm. The results of proposed hybrid DE algorithm are quite convincing in fast error convergence and stable classification accuracy than hybrid GA algorithm.

Finally, from the experiments, we can conclude that DE-BP takes less CPU time, with maintaining higher training accuracy than other three algorithms. From the experiments, it can also see that DE-BP algorithm has smooth MSE and accuracy rate than other algorithms. In future research works, we shall focus on how to extend this work to solve more real world problems.

\section{REFERENCES}

[1] J. Ilonen, J. K. Kamarainen, and J. Lampinen: Differential evolution training algorithm for feed-forward neural networks, Neural Processing Letters, 17:93-105, 2003.

[2] P. P. Sarangi, B. Majhi and M. Panda, "Performance Analysis of Neural Networks Training using Real Coded Genetic 
Algorithm", International Journal of Computer Applications 51(18):30-36, 2012

[3] H. Hasanrkc, HasanBal, "Comparing performances of backpropagation and genetic algorithms in the data classification", Expert Systems with Applications, Volume 38, Issue 4,Pages 3703-3709, 2011.

[4] Zhang, G., "Neural networks for classification: a survey", IEEE Transactions on Systems, Man, and Cybernetics, Part C 30(4): 451-462, 2000.

[5] S. B. Kotsiantis, "Supervised Machine Learning: A Review of Classification Techniques", Informatica31 249-268, 2007

[6] Curry B, Morgan P. Neural networks: a need for caution. Omega, International Journal of Management Sciences, 1997

[7] D. J. Montana and L. Davis, Training Feedforward Neural Networks using Genetic Algorithms, Proceedings of the Third International Conference on Genetic Algorithms, Morgan Kaufmann, San Mateo, CA, 379-384,1989

[8] Sexton, R., Dorsey, R., and Johanson, J., "Optimization of Neural Networks: A Comparative Analysis of the Genetic Algorithm and Simulated Annealing", European Journal of Operational Research, volume 114, issue 3,page 589-601,1999.

[9] Sexton, R., Dorsey, R., and Johanson, J., "Toward a Global Optimization for Neural Networks:, A Comparison of the Genetic Algorithm and Backpropagation", forthcoming in Decision Support Systems.

[10] Gupta, J. N. D., and Sexton, R. S. Comparing backpropagation with a genetic algorithm for neural network training. Omega, 27, 679-684.

[11] X. Yao, Evolving artificial neural networks, Proc. IEEE 87 (9), 1423-1447, 1999.

[12] Werbos P. The roots of the backpropagation: from ordered derivatives to neural networks and political forecasting. New York: John Wiley and Sons, Inc, 1993

[13] D.E. Rumelhart, G.E. Hinton, R.J. Williams, Learning representations by back-propagating errors, Nature 323 533-536, 1986

[14] Simon Haykin, "Neural Networks: A comprehensive foundation," Pearson Education Asia, Seventh Indian Reprint, 2004.

[15] D. Whitley, "Applyong Genetic Algorithms to Neural Network Problems," International Neural Network Society pp.230, 1988.

[16] Prechelt, L.: Proben1, "A Set of Neural Network Benchmark Problems and Benchmarking Rules". Technical Report 21, FakultatfurInformatik University at Karlsruhe, 76128 Karlsruhe, Germany, 1994.

[17] Schaffer J. D., D. Whitley, and L. J. Eshelman, "Combinations of Genetic Algorithms and Neural Networks: A Survey of the State of the Art, " Proceedings of the IEEE Workshop on Combinations of Genetic Algorithms and Neural Network.

[18] C. Zhang, H. Shao. and Y. Li. "Panick swarm optimization for evolving artificial neural network", Procccdings of the IEEE Intcrnational Conference 011 System, Man, and Cybcmetics. vol. 4, pp.24x7-2490, 2000.

[19] Zhang, J. R., Zhang, J., Lok, T. M., and Lyu, M. R. (2007). A hybrid particle swarm optimization-back-propagation algorithm for feedforward neural network training. Applied Mathematics and Computation 185, 1026 - 1037.

[20] Zhang, C., and Shao, H., An ANN's evolved by a new evolutionary system and its application. In Proc. of the 39th IEEE conf. on decision and control. vol. 4, pp. 3562 - 3563, 2000.
[21] M. Sellcs and B. Rylander, "Neural network learning using particle swarm optimization", Advances in Information Science and SoftComputing. pp. 224-226, 2002

[22] R. Storn, and K. Price, "Differential Evolution-A Simple and Efficient Heuristic for Global Optimization over Continuous Spaces, "Journal of Global Optimization, Vol. 11, pp. 341359, 1997.

[23] Adam Slowik, and Michal Bialko, "Training of Artificial Neural Networks Using Differential Evolution Algorithm", Krakow, Poland, May 25-27, 2008

[24] UCI repository of machine learning databases, Department of Information and Computer Sciences, University of California, Irvine, http://www.ics.uci.edu/ mlearn/MLRepositor. 